\title{
Asymptomatic Rhabdomyoma of the Larynx: Case Report and Review of the Literature
}

\author{
Ahmed HS1, Parikh AS ${ }^{2}$, Srikanth P1, Tjoa T3 , \\ Faquin $\mathrm{WC}^{4}$ and Lin $\mathrm{DT}^{2 *}$ \\ ${ }^{1}$ Harvard Medical School, USA \\ ${ }^{2}$ Department of Otolaryngology, Massachusetts Eye and \\ Ear Infirmary, USA \\ ${ }^{3}$ Department of Otolaryngology, University of California \\ Irvine, USA \\ ${ }^{4}$ Department of Pathology, Massachusetts Eye and Ear \\ Infirmary, USA \\ *Corresponding author: Lin DT, Department of \\ Otolaryngology, Massachusetts Eye and Ear Infirmary, \\ 243 Charles Street, Boston, MA 02114, USA
}

Received: January 09, 2017; Accepted: February 13, 2017; Published: February 17, 2017

\begin{abstract}
Rhabdomyoma of the head and neck is an uncommon tumor. Tumor occurrence in the larynx is particularly rare, with only 53 reported cases. We describe the case of a 59-year-old woman with an asymptomatic, incidentally discovered adult type rhabdomyoma of the left aryepiglottic fold, successfully treated with endoscopic resection. We also conduct a comprehensive review of the literature, including operative management and surgical outcomes. Of 53 cases reviewed, the mean age at diagnosis was 33.8 years and a 2.4:1 male predominance was observed. The most common presenting symptom was hoarseness, and the most common site of origin was the true vocal fold. Endoscopic and open resection were used at similar rates, and there were no obvious differences in patient characteristics by surgical approach. However, a higher recurrence rate and shorter time to recurrence were observed with endoscopic resection, as compared with open resection. Thus, we suggest the potential downside of endoscopic management that must be balanced with the potential for significantly lower morbidity and ease of re-resection when considering the appropriate surgical approach for a given patient.
\end{abstract}

Keywords: Larynx; Rhabdomyoma; Laryngeal neoplasms

\section{Introduction}

Rhabdomyomas are extremely rare benign neoplasms of striated muscle and comprise less than $2 \%$ of all skeletal muscle tumors [1]. Most extracardiac rhabdomyomas occur in the head and neck, specifically the oropharynx, larynx, pharyngeal constrictor muscles, submandibular region, base of the tongue, and less commonly lip, lateral neck, soft palate, uvula, cheek, and orbit [2-6].

Rhabdomyomas of the head and neck are slow-growing and wellcircumscribed; malignant transformation is rare [4,7]. Treatment generally involves surgical excision of the lesion, preventing invasion of surrounding tissues and obstruction of the airway or esophageal inlet. Although treatment is typically curative, these lesions do occasionally recur. Histologically, rhabdomyomas are divided into two types: neoplastic and hamartoma [8]. Neoplastic rhabdomyomas are further classified into adult, fetal, and genital (or vaginal) subtypes [8]. Adult type rhabdomyomas are characterized by sheets of closelypacked polygonal vacuolated (glycogen-containing) cells with granular eosinophilic cytoplasm, with occasional cross-striations and prominent nucleoli $[2,3]$. Fetal cellular type is characterized by immature skeletal muscle elements at varying stages of differentiation [2], while genital cellular type appears as a mixture of fibroblast-like cells with clusters of mature cells with cross-striations and collagenand mucoid-rich matrix [3].

Clinically, adult type rhabdomyomas occur in the soft tissues of the head and neck in 70 to $93 \%$ of cases; the fetal subtype is most prevalent in children, and the genital (also known as vaginal) type is typically a polypoid mass found in the vagina and vulva of middle-aged women $[3,4,6]$. The most common presenting symptoms for the adult type are hoarseness, dyspnea, and dysphagia [3,4]. Fetal type rhabdomyomas present with obstructive and constrictive oropharyngeal symptoms, and the genital type is typically asymptomatic but presents with dyspareunia $[3,9]$.

Here, we describe the case of an incidentally discovered adult rhabdomyoma of the larynx in a 59-year-old woman. We also systematically review 53 cases of laryngeal rhabdomyoma reported in the literature, which to our knowledge, has not been done previously.

\section{Case Presentation}

The patient is 59 -year-old woman with a 40 pack year smoking history who presented to her primary care physician for routine care. Given her extensive smoking history, a screening chest CT was ordered and demonstrated a left upper lobe lung mass. Subsequent PET-CT showed this lung mass, as well as an incidental $3.2 \mathrm{~cm}$ left supraglottic laryngeal mass, and the patient was referred to otolaryngology. Fiberoptic laryngoscopy showed a large obstructing left arytenoid mass with normal overlying mucosa. Dedicated neck CT showed a well-circumscribed, oval, left supraglottic mass measuring $3.3 \times 2.8 \times 1.8 \mathrm{~cm}$, centered in the left piriform sinus and along the left aryepiglottic fold, without involvement of the true vocal folds (Figure 1).

The patient underwent direct laryngoscopy with biopsy and at that time, and an elective tracheotomy was performed due to concern for airway obstruction from the mass. The biopsy showed large polygonal tumor cells, with immunohistochemistry strongly positive for desmin and negative for keratin, S-100, Sox-10, NSE, and CD68, consistent with a diagnosis of adult rhabdomyoma. The patient underwent endoscopic surgical resection, with a combination of laryngeal microscissors, OmniGuide $\mathrm{CO}_{2}$ laser, and microdebrider (Figure 2). A complete resection was achieved, with the area of attachment to 


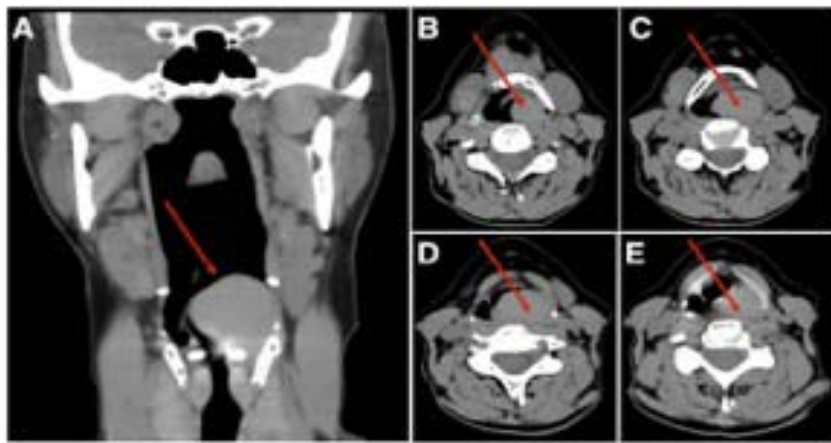

Figure 1: CT neck showing left supraglottic mass on coronal $(A)$ and axial (B-E) sections.

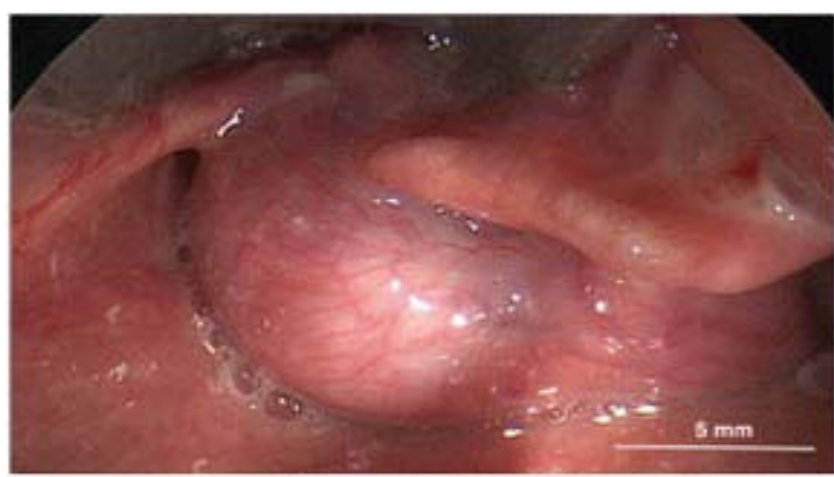

Figure 2: Intra-operative image showing large, well-circumscribed mass arising from left aryepiglottic fold.

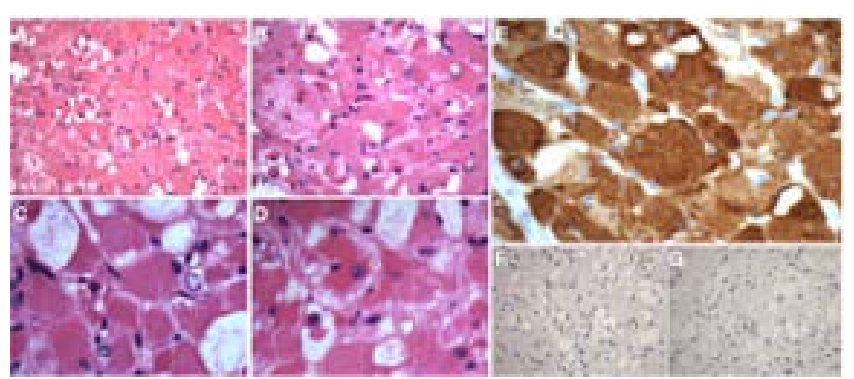

Figure 3: Hematoxylin and eosin staining of adult rhabdomyoma showing large polygonal vacuolated cells with eosinophilic cytoplasm (A, B), with occasional cross-striations (C) and prominent nucleoli (D). Immunostating of adult rhabdomyoma showing positive desmin immunoreactivity $(E)$ and negative synaptophysin (F) and S-100 (G) and immunoreactivity.

the left aryepiglottic fold free of gross disease. Final pathology was consistent with the initial biopsy specimen (Figure 3). The patient had no evidence of tumor recurrence at a 9 month follow up visit. Regarding her lung mass, she also underwent left upper lobectomy, which revealed stage I non-small cell lung cancer.

\section{Discussion and Conclusion}

We describe the case of an asymptomatic, incidentally-discovered rhabdomyoma of the supraglottic larynx, arising from the left aryepiglottic fold. We also perform a comprehensive review the literature using the MEDLINE database as indexed by PubMed. The MEDLINE database was searched using the terms "rhabdomyoma

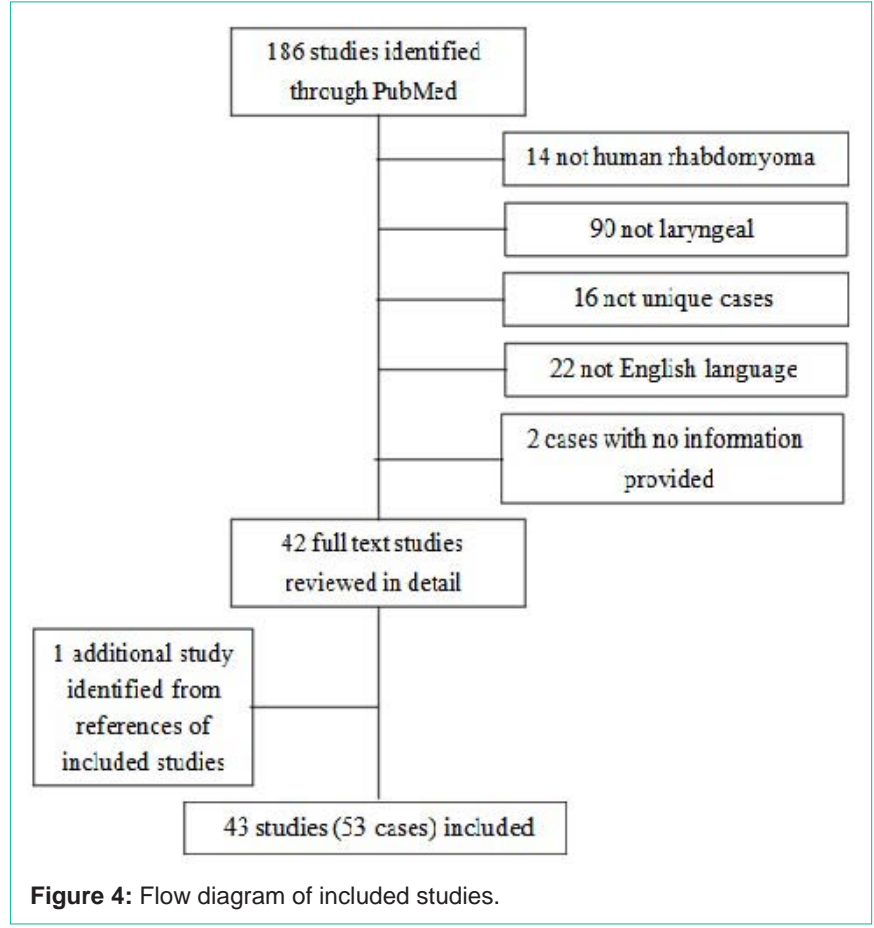

larynx," "laryngeal rhabdomyoma," and "rhabdomyoma head neck." A total of 186 entries were screened by title and abstract. Articles were excluded if they were not human rhabdomyoma (14), not laryngeal (90), not unique cases (13), or not English-language (22). In addition, one case was added based on references from articles obtained from the above searches [2]. A total of 53 cases were included (Figure 4) (Table 1).

Patients with laryngeal rhabdomyoma are most commonly middle-aged men presenting with hoarseness [7]. Other presenting symptoms include dysphagia [7], dyspnea [10], dysphonia [11], and rarely stridor [11], aspiration [12], and airway obstruction [13]. Mean age at diagnosis was 33.8 years (range 1.25 to $82 \mathrm{yrs}$ ), with a 2.4:1 male predominance. $40 / 53$ cases $(75 \%)$ were adult rhabdomyomas, $11 / 53$ (21\%) were fetal type, $2 / 53$ (4\%) did not report a type. The most common subsites were the true vocal fold (20/53 cases, $38 \%)$, false vocal fold (7/53 cases, 13\%), and the aryepiglottic fold (5/53 cases, 9\%).

On biopsy, histopathology typically demonstrates large closelypacked polygonal cells that often have a spiderweb-like appearance $[14,15]$. Immunohistochemically, rhabdomyomas stain positively for myoglobin, desmin, and myo-D1, with occasionally positive musclespecific actin. Immunoreactivity for smooth muscle actin, S-100 protein, vimentin, Leu-7 and cytokeratin are typically negative. There is typically no immunoreactivity for epithelial markers, chromogranin, synaptophysin, glial fibrillary acidic protein, or CD68 (KP-1), and the expression of proliferation marker ki-67 is low, consistent with the indolent, slow-growing nature of these tumors. Electron microscopy typically shows thin and thick myofilaments with Z-band material $[14,15]$. An accurate diagnosis can be made due to the characteristic microscopic appearance and immunohistochemical staining. Our patient's histopathology and immunostaining are consistent with the diagnosis of adult type rhabdomyoma. 
Table 1: Laryngeal rhabdomyomas previously described in literature.

\begin{tabular}{|c|c|c|c|c|c|c|}
\hline Age/ Sex & Site & Symptom(s) & Treatment & Type & $\begin{array}{l}\text { Size } \\
(\mathrm{cm})\end{array}$ & Follow-up/Outcome \\
\hline $23 \mathrm{M}$ & TVF & Hoarseness & Endoscopic excision & Fetal & 1.5 & No recurrence after $1 \mathrm{yr}$ \\
\hline $39 F$ & FVF to subglottis & Hoarseness & Laryngofissure & NS & 3 & No follow up reported \\
\hline $82 \mathrm{M}$ & TVF & Hoarseness & None (found at autopsy) & Adult & 1 & No recurrence at $20 \mathrm{yr}$ \\
\hline $48 \mathrm{M}$ & TVF & Hoarseness & Endoscopic excision & Adult & 1 & No recurrence at $1 \mathrm{yr}$ \\
\hline $55 \mathrm{M}$ & FVF to subglottis & Hoarseness & Laryngofissure & Adult & 5 & No follow up reported \\
\hline $36 \mathrm{~F}$ & Ventricle & Hoarseness & Laryngofissure & Adult & 1.5 & No follow up reported \\
\hline $50 \mathrm{M}$ & Interarytenoid region & $\begin{array}{l}\text { Hoarseness, } \\
\text { dysphonia }\end{array}$ & Endoscopic excision & Fetal & 1.5 & No recurrence at $1 \mathrm{yr}$ \\
\hline $52 \mathrm{~F}$ & FVF & Hoarseness & Endoscopic excision & Adult & 0.5 & No recurrence after 7 mo \\
\hline $39 \mathrm{M}$ & TVF & Hoarseness & Endoscopic excision & Adult & 0.5 & Local resections for recurrences at 2, 7, 11 mo \\
\hline $55 \mathrm{M}$ & FVF & Not reported & Endoscopic excision & Adult & 2.5 & No recurrence at $2 \mathrm{yr}$ \\
\hline $16 \mathrm{M}$ & $\begin{array}{l}\text { TVF, thyroid } \\
\text { cartilage }\end{array}$ & Airway obstruction & Total laryngectomy & Adult & 2 & No recurrence at several yr \\
\hline $76 \mathrm{~F}$ & TVF & Hoarseness & Endoscopic excision & Adult & 0.75 & No recurrence at $2 \mathrm{yr}$ \\
\hline $53 \mathrm{M}$ & TVF & Hoarseness & Open excision & Fetal & NS & No recurrence at $3.5 \mathrm{yr}$ \\
\hline $65 \mathrm{~F}$ & TVF & Hoarseness & Open excision & Fetal & NS & No recurrence at $1.5 \mathrm{yr}$ \\
\hline $58 \mathrm{M}$ & $\begin{array}{l}\text { AE fold, pyriform } \\
\text { sinus }\end{array}$ & Dysphagia, globus & Transhyoidpharyngotomy & Adult & 3 & No recurrence at $2 \mathrm{yr}$ \\
\hline $34 \mathrm{~F}$ & Anterior TVF & Hoarseness & Endoscopic excision & Fetal & 3 & $\begin{array}{l}\text { Two recurrences; lateralpharyngotomy then } \\
\text { supraglotticlaryngectomy at } 7 \mathrm{mo} \text {, no recurrence at } 3 \mathrm{yr}\end{array}$ \\
\hline $60 \mathrm{M}$ & Ventricle & Not reported & Unspecified & Adult & 15 & No follow up reported \\
\hline $31 \mathrm{M}$ & FVF & Hoarseness & Lateral thyrotomy & Fetal & 3 & No recurrence at $1 \mathrm{yr}$ \\
\hline $78 \mathrm{~F}$ & Posterior TVF & Hoarseness & Endoscopic excision & Fetal & 0.6 & No recurrence at $2 \mathrm{yr}$ \\
\hline $52 \mathrm{M}$ & TVF & Hoarseness & Lateral pharyngotomy & Adult & 1.5 & No recurrence at $18 \mathrm{mo}$ \\
\hline $29 M$ & FVF & Hoarseness & Unspecified & Fetal & 1.2 & No recurrence at $2 \mathrm{yr}$ \\
\hline $51 \mathrm{~F}$ & Arytenoid & Dyspnea, dysphagia & Lateral pharyngotomy & Adult & 4 & Resection for recurrence $12 \mathrm{yr}$, no recurrence at $2 \mathrm{yr}$ \\
\hline $15 \mathrm{mo} \mathrm{F}$ & Subglottis & Dysphagia, stridor & Open excision & Adult & 2 & No recurrence after 6 mo \\
\hline $32 \mathrm{M}$ & Larynx & Not reported & Unspecified & Adult & NS & No follow up reported \\
\hline $59 \mathrm{M}$ & Larynx & Hoarseness & Unspecified & Adult & NS & No recurrence at $5 \mathrm{yr}$ \\
\hline $59 M$ & $\begin{array}{l}\text { BOT, phayrnx, } \\
\text { larynx }\end{array}$ & Airway obstruction & Unspecified & Adult & NS & No recurrence at $10 \mathrm{yr}$ \\
\hline $60 \mathrm{M}$ & Larynx & Airway obstruction & Unspecified & Adult & 1.5 & No recurrence at $2 \mathrm{yr}$ \\
\hline $54 \mathrm{M}$ & Larynx, hypopharynx & Airway obstruction & Unspecified & Adult & NS & No recurrence at $2 \mathrm{yr}$ \\
\hline $56 \mathrm{~F}$ & Interarytenoid region & Dyspnea, dysphagia & Endoscopic excision & Adult & 2.5 & $\begin{array}{l}\text { Recurrence resected, date unknown, no recurrence at } \\
\qquad 1 \mathrm{yr}\end{array}$ \\
\hline $31 \mathrm{~F}$ & TVF & Dysphonia & Unspecified & Adult & NS & No follow up reported \\
\hline $51 \mathrm{M}$ & Ventricle & Hoarseness & Hemilaryngectomy & Adult & 7.5 & No recurrence at $1 \mathrm{yr}$ \\
\hline $64 \mathrm{M}$ & AE fold & Asymptomatic & Lateral pharyngotomy & Adult & 5 & $\begin{array}{l}\text { Lateral pharyngotomy for recurrence at } 2 \mathrm{mo} \text {, } \\
\text { contralateral pharyngotomy for recurrence at } 7 \mathrm{yr}\end{array}$ \\
\hline $69 \mathrm{~F}$ & TVF & Hoarseness & Endoscopic excision & Adult & NS & No recurrence at 6 mo \\
\hline $39 \mathrm{M}$ & AE fold, TVF & Dysphagia, aspiration & Unspecified & Adult & NS & No follow up reported \\
\hline $66 \mathrm{M}$ & Arytenoid & $\begin{array}{l}\text { Hoarseness, } \\
\text { dysphagia }\end{array}$ & Open excision & Adult & NS & No recurrence \\
\hline $81 \mathrm{M}$ & TVF & $\begin{array}{l}\text { None (incidental } \\
\text { finding) }\end{array}$ & Laryngectomy for SCC & Adult & 0.04 & No follow up reported \\
\hline $79 \mathrm{M}$ & FVF & Hoarseness & Open excision & Adult & 2 & No recurrence at 6 weeks \\
\hline $69 \mathrm{M}$ & Epiglottis & None & Unspecified & Adult & 2.8 & No follow up reported \\
\hline $66 \mathrm{M}$ & Arytenoid & $\begin{array}{l}\text { Dysphagia, } \\
\text { obstruction }\end{array}$ & Endoscopic excision & Adult & 2 & No recurrence at $18 \mathrm{mo}$ \\
\hline
\end{tabular}




\begin{tabular}{|c|c|c|c|c|c|c|}
\hline $72 \mathrm{~F}$ & AE fold & Hoarseness, globus & Transoral laser excision & Adult & 3.5 & No follow up reported \\
\hline $76 \mathrm{M}$ & Arytenoid & $\begin{array}{c}\text { Hoarseness, } \\
\text { dysphagia }\end{array}$ & Laser debulking & Adult & 3.5 & Recurrences at 5,9 mo \\
\hline $68 \mathrm{M}$ & Supraglottis & Dysphagia, globus & Open excision & Adult & 5.5 & No recurrence at 10 mo \\
\hline NS & Glottis & Dysphonia & Endoscopic excision & NS & NS & No follow up reported \\
\hline $67 \mathrm{~F}$ & Supraglottis & Hoarseness, dyspnea & Hemilaryngectomy & Adult & 3 & No recurrence at 16 mo \\
\hline $55 \mathrm{M}$ & Supraglottis & $\begin{array}{c}\text { Hoarseness, } \\
\text { dysphagia }\end{array}$ & Endoscopic excision & Adult & 8.5 & Recurrence at 3 mo \\
\hline $35 \mathrm{M}$ & Supraglottis & Hoarseness & Endoscopic excision & Adult & NS & No recurrence at 15 mo \\
\hline $42 \mathrm{M}$ & TVF & Hoarseness & Endoscopic excision & Fetal & 0.5 & No follow up reported \\
\hline $11 \mathrm{M}$ & Supraglottis, TVF & Dysphonia, stridor & Endoscopic excision & Fetal & 1.6 & No recurrence at 5 yr \\
\hline $75 \mathrm{M}$ & TVF & Hoarseness & Endoscopic excision & Adult & 2.2 & No recurrence at 1 yr \\
\hline $59 F \dagger$ & AE fold & None & Endoscopic excision & Adult & 3.3 & No recurrence at 9 mo \\
\hline
\end{tabular}

TVF: True Vocal Fold; AE: Aryepiglottic; FVF: False Vocal Fold; NS: Not Stated; yr: year; mo: months

${ }^{\dagger}$ Current report

Patients with laryngeal rhabdomyoma are generally treated surgically. Of the cases reported in the literature, $18 / 53$ patients (34\%) had open resections, while $22 / 53$ patients (42\%) had endoscopic resections; there were 13 additional cases for which the surgical details were not reported. Patients who underwent open resection tended to have slightly larger lesions (average size $2 \mathrm{~cm} v s .1 .6 \mathrm{~cm}$ for lesions resected endoscopically), but this difference was not statistically significant. There was no obvious difference in resection technique by age, subsite, or presenting symptom. Of patients who underwent open resection, 2/18 (11\%) recurred, while of those who underwent endoscopic resection, 5/22 (23\%) recurred. Moreover, the average time to recurrence in patients treated with open surgery was 6.1 years, while in patients treated endoscopically, it was 4.3 months.

Although this sample is small and heterogeneous, these data suggest anecdotally that there may be a higher recurrence rate with endoscopic resection than with open resection. However, this consideration must be balanced with the increased morbidity of an open resection, as well as the relative ease of repeat endoscopic resection when necessary, particularly given the benign nature of these lesions. Moreover, although these lesions do occasionally cause airway obstruction they typically present with other symptoms first and may be readily caught at an early stage with routine surveillance. Thus, endoscopic resection should still be considered when possible but given the potentially increased rate of and accelerated time to recurrence, frequent surveillance should be considered, particularly in the early postoperative period.

\section{References}

1. Papaspyrou G, Werner JA, Roeßler M, Devaney KO, Rinaldo A, Ferlito A Adult rhabdomyoma in the parapharyngeal space: report of 2 cases and review of the literature. Am J Otolaryngol. 2011; 32: 240-246.

2. Imperatori CJ. Rhabdomyoma of the larynx. Report of a case. The Laryngoscope. 1933; 43: 945-948.
3. Enzinger FM, Weiss SW. Rhabdomyoma. In: Enzinger FM, Weiss SW, eds. Soft tissue tumors, 3rd ed. St. Louis: Mosby; 1995; 523-536.

4. Kapadia SB, Meis JM, Frisman DM, Ellis GL, Heffner DK, Hyams VJ. Adult rhabdomyoma of the head and neck: a clinicopathologic and immunophenotypic study. Hum Pathol. 1993; 24: 608-617.

5. Bastian BC, Bröcker EB. Adult rhabdomyoma of the lip. Am J Dermatopathol. 1998; 20: 61-64.

6. Knowles DM, Jakobiec FA. Rhabdomyoma of the orbit. American journal of ophthalmology. 1975; 80: 1011-1018.

7. Koutsimpelas D, Weber A, Lippert BM, Mann WJ. Multifocal adult rhabdomyoma of the head and neck: a case report and literature review. Auris Nasus Larynx. 2008; 35: 313-317.

8. Leon Barnes, John W. Eveson, Peter Reichart DS. WHO Classification of Tumours. Pathol Genet Head Neck Tumours. 2005; 209-281.

9. Montgomery EA. Biopsy interpretation of the gastrointestinal tract mucosa. Lippincott Williams \& Wilkins. 2006.

10. Pichi B, Manciocco V, Marchesi P, Pellini R, Ruscito P, Vidiri A, et al. Rhabdomyoma of the parapharyngeal space presenting with dysphagia. Dysphagia. 2008; 23: 202-204.

11. Elawabdeh N, Sobol S, Blount AC, Shehata BM. Unusual presentation of extracardiac fetal rhabdomyoma of the larynx in a pediatric patient with tuberous sclerosis. Fetal Pediatr Pathol. 2013; 32: 43-47.

12. Liang GS, Loevner LA, Kumar P. Laryngeal rhabdomyoma involving the paraglottic space. Am J Roentgenol. 2000;174:1285-1287.

13. Jensen K, Swartz K. A rare case of rhabdomyoma of the larynx causing airway obstruction. Ear Nose Throat J. 2006; 85: 116-118.

14. Weiss SW, Goldblum JR. Osseous soft tissue tumors. Weiss SW, Goldblum JR. Enzinger and Weiss Soft Tissue Tumors. 2001.

15. Wenig BM. Atlas of head and neck pathology. Elsevier Health Sciences. 2015.
Ann Hematol Oncol - Volume 4 Issue 2 - 2017 ISSN : 2375-7965 | www.austinpublishing group.com Lin et al. @ All rights are reserved
Citation: Ahmed HS, Parikh AS, Srikanth P, Tjoa T, Faquin WC and Lin DT. Asymptomatic Rhabdomyoma of the Larynx: Case Report and Review of the Literature. Ann Hematol Oncol. 2017; 4(2): 1135. 